\title{
Research Status and Trend of Unstructured Information Processing based on Cognition
}

\author{
Shixin Fan ${ }^{1, *}$, Gang Sun², Lin Qiao ${ }^{3}$, Ying Liu ${ }^{3}$, Jiang Chang ${ }^{4}$, Zhuang Li $^{5}$ \\ ${ }^{1}$ Personnel Director Department, State Grid Liaoning Electric Power Supply Co., Ltd: Shenyang 110006, \\ China \\ ${ }^{2}$ Human Resource Department, State Grid Liaoning Electric Power Supply Co., Ltd: Shenyang 110006 China \\ ${ }^{3}$ Operation \& Maintenance Center, State Grid Liaoning Information and Communication Company: Shenyang \\ 110006 China \\ ${ }^{4}$ Logistics Department, State Grid Liaoning Electric Power Supply Co., Ltd: Shenyang 110006 China \\ ${ }^{5}$ Fujian Yirong Information Technology Co., Ltd: Fuzhou, 350100 China \\ *Corresponding Author email: fsx@ln.sgcc.com.cn
}

Keywords: Unstructured information processing; environment perception; machine learning

\begin{abstract}
Unstructured data have complex features such as ultra-high dimension, nonlinearity, and interpretation of information through subjects, which lead to the difficulties in management, processing and application of unstructured Information. On this basis, this paper illustrates the research status and research trend of unstructured information processing from the perspective of human cognition, expecting to bring some inspiration to scholars in the field.
\end{abstract}

\section{Introduction}

With the advent of the information age and the rapid development of big data and other new technologies, the strategic resource attributes of information have received more attention from various countries, and the widespread unstructured data has become the focus of academic research. The representation and modeling of unstructured information, mathematical modeling and knowledge reasoning, comprehensive utilization and behavior decision are all representatives of relevant research. This paper studies the research status and trend of unstructured information processing in order to thoroughly understand the relevant research.

\section{Research status of unstructured information processing}

\subsection{Research progress}

As computers and smart phones have become popular among the people of various countries, the growth rate of unstructured information is gradually increased, and the research of unstructured information processing based on cognitive science principle and neurophysiology has become the focus of attention at home and abroad. In recent years, domestic and foreign scholars have carried out a great deal of research on unstructured information processing. The unstructured information management architecture (UIMA) constructed by the IBM Company of the United States is a representative. Figure 1 is a schematic diagram of an unstructured information management framework, the development of which marks that unstructured information can be used through a new approach. In recent years, Chinese scholars' research on knowledge management system, information retrieval system, database monitoring document analysis based on unstructured information management architecture has also proved its practical value. In addition to IBM Company, other companies such as Oracle and Cogno also developed some systems for unstructured information processing. However, based on comprehensive analysis of these systems, it can be found 
that there is sufficient development potential in unstructured information processing despite of the rapid progress at home and abroad in recent years ${ }^{[1]}$.

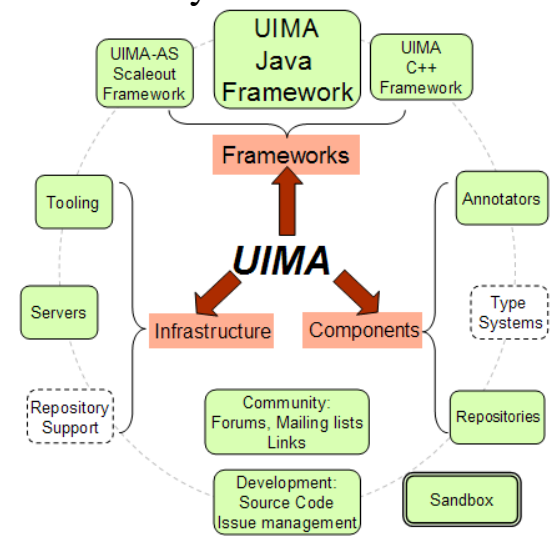

Figure 1 Unstructured information management framework

\subsection{Relevant research fields}

Environmental perception, machine learning, knowledge reasoning and integrated integration have become the focus of academic research on unstructured information processing in recent years. The relevant research is derived from the perspective of human cognition, and the characteristics of research in various fields are as follows: (1) Environmental perception: Environmental perception research focuses on the reconstruction of real physical scene. "User oriented multidimensional information integrated environment”, PAL program and intelligent driving are all research achievements in this field in recent years. Table 1 briefly introduces the key technologies of intelligent driving environment perception so as to better understand environment perception. (2) Machine learning. Machine learning is also an important research field in unstructured information processing. The core of the research is to provide solid foundation support for unstructured data processing. Neural network technology, data clustering and characteristic subspace analysis all belong to the typical research results. (3) Knowledge reasoning: As an important research field, knowledge reasoning can also provide strong support for unstructured information processing. It is essentially an information processing method that conforms to human reasoning rules. The processing of uncertain and incomplete information is the strength of knowledge reasoning. Table 2 shows a reasoning service implementation method based on a UIMA enterprise knowledge management system, so that the Application value of knowledge reasoning in this field can be directly perceived. (4) Integrated integration: Integrated integration refers to a variety of information processing methods and the comprehensive technology application methods. In recent years, the development of various integrated systems facilitates the close connection of the processing methods, which can better serve unstructured information processing ${ }^{[2]}$.

Table 1 Key technologies of intelligent driving environment perception

\begin{tabular}{cc}
\hline Key technologies & Contents \\
\hline Sensor calibration & $\begin{array}{c}\text { Laser radar calibration, camera calibration and joint } \\
\text { calibration of camera and laser radar; }\end{array}$ \\
$\begin{array}{c}\text { Structured road } \\
\text { detection }\end{array}$ & $\begin{array}{c}\text { Straight road detection, curve detection, and preprocessing } \\
\text { of detected image under complex environment; } \\
\text { Self-supervised sample acquisition, online learning } \\
\text { Unstructured road } \\
\text { detection }\end{array}$ \\
$\begin{array}{c}\text { Target detection in } \\
\text { algorithm, dynamic training sample library and prediction } \\
\text { model training; }\end{array}$ \\
\hline
\end{tabular}


Table 2 Reasoning service realization method of knowledge management system in a UIMA enterprise (Part)

\begin{tabular}{ccc}
\hline Method introduction & $\begin{array}{c}\text { Returned } \\
\text { value }\end{array}$ & Method signature \\
\hline $\begin{array}{c}\text { Execute reasoning hierarchy } \\
\text { structure }\end{array}$ & String & start Reason() \\
$\begin{array}{c}\text { Acquire the subclasses } \\
\text { Save the hierarchy structure }\end{array}$ & $\begin{array}{c}\text { ArrayList } \\
\text { String }\end{array}$ & $\begin{array}{c}\text { getSubclasses(StringclsName) } \\
\text { saveResult(ArrayListclassNameList) }\end{array}$ \\
\hline
\end{tabular}

\section{Research trend of unstructured information processing}

Based on a brief understanding of the research status of unstructured information processing under cognition, this paper summarizes the following three aspects of unstructured information processing combined the research results at home and abroad, expecting to bring some inspiration to scholars in the industry.

\subsection{Semi-structured data conversion}

For the research trend of semi-structured data conversion as unstructured information processing, Oracle XML DB of Oracle Company is the representative. The expansion of database storage function through native XML storage is an intuitive presentation of the research trend, which satisfy the demands of full-text index and content extraction of unstructured data and support the unstructured information processing. In addition, the Hadoop environment is also an important application direction of semi-structured data conversion. Figure 2 shows a semi-structured data conversion full text retrieval system based on Hadoop environment. Figure 3 is the diagram of the HDFS distributed file system read-write data provided by the Hadoop environment.

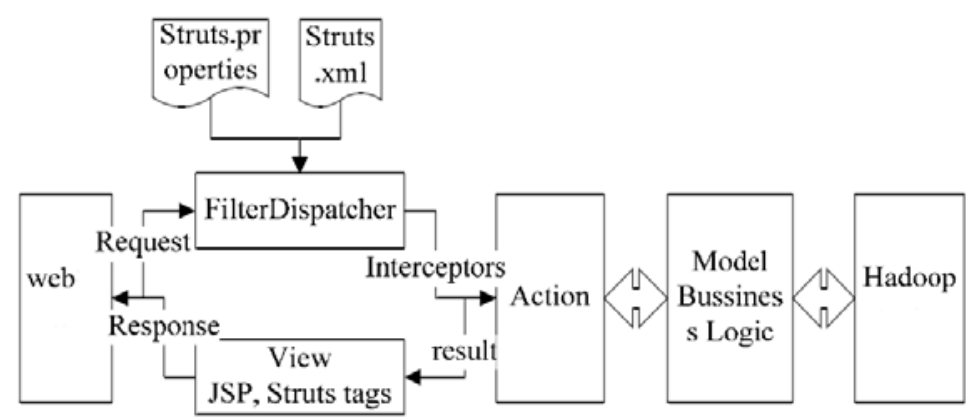

Figure 2 semi-structured data conversion full text retrieval system based on Hadoop environment.

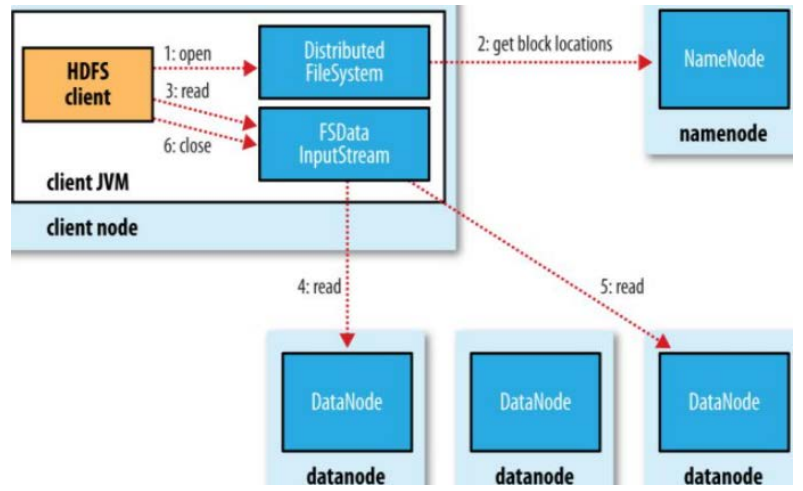

Figure 3 Diagram of HDFS distributed file system read-write data

\subsection{Application of big data and cloud computing technology}

With the arrival of large data and cloud ear, the research on the application field of large data technology has attracted increasing attention from the academic circle. The application value of large 
data and cloud computing technology in the field of unstructured information processing has also been highly concerned, which is represented by the studies of large data mass information storage architecture developed by the academic community in recent years. In general, cloud service provides comprehensive services to unstructured data processing based on big data technology, such as data mining, data storage, full-text retrieval, video transcoding and image recognition. Ali cloud, Qiniu cloud and Amazon cloud are typical representatives. Table 3 shows the characteristics of the services provided by Ali cloud and Qiniu cloud.

Table 3 Characteristics of the services provided by Ali cloud and Qiniu cloud

\begin{tabular}{cc}
\hline $\begin{array}{c}\text { Cloud } \\
\text { service } \\
\text { provider }\end{array}$ & Service characteristics \\
\hline Ali Cloud & $\begin{array}{c}\text { Provide one-stop data services, including data storage, data acquisition } \\
\text { and data processing. Users do not need to consider security and } \\
\text { implementation issues. Data visualization, audio and video transcoding, } \\
\text { and image recognition all belong to the specific functions of Ali cloud. } \\
\text { Piniu }\end{array}$ \\
$\begin{array}{c}\text { Provide safe data storage service. Online audio and video/streaming } \\
\text { media processing service, and data monitoring service are the } \\
\text { characteristics of the service provided by Qiniu Cloud. }\end{array}$ \\
\hline
\end{tabular}

\subsection{Multimode heterogeneous information fusion}

In addition to the above two aspects, multimode heterogeneous information fusion is also one of the research trends of unstructured information processing. Biological experiment results play important roles in this field. Table 4 shows the hot word screening results in a video website implemented through multimode heterogeneous information fusion, which proves the high development potential of multi-mode heterogeneous information fusion.

Table 4 Hot word screening results in a video website (Part)

\begin{tabular}{ccc}
\hline ID & Keywords & Number \\
\hline 1 & bush shoe & 210 \\
2 & Michael Jackson & 895 \\
3 & brad pitt & 489 \\
4 & Miley Cyrus & 998 \\
\hline
\end{tabular}

\section{Conclusion}

To sum up, unstructured information processing based on cognition has great development potential. On this paper, semi-structured data conversion, the application of large data and cloud computing technology and multimode heterogeneous information fusion discussed in this paper prove the practical value of the research. Therefore, this paper can be used for reference in the theoretical research and practical exploration of the related fields.

\section{References}

[1] Mukherjee S, Bala P K. Gender classification of microblog text based on authorial style [J]. Information Systems and e-Business Management, 2016, 15(1):1-22.

[2] Jr W A, Keiser N L, Doverspike D. An Information-Processing-Based Conceptual Framework of the Effects of Unproctored Internet-Based Testing Devices on Scores on Employment-Related Assessments and Tests [J]. Human Performance, 2017(1):1-32. 